\title{
Temperature Measurement of an Inclined Cylinder Using Optical Interferometry
}

\author{
J.R. Zhu \\ School of Materials Science and Engineering \\ Wuhan University of Technology \\ China \\ Hubei Collaborative Innovation Center for High-efficiency \\ Utilization of Solar Energy \\ Hubei University of Technology \\ China \\ J.M. Dai \\ Hubei Collaborative Innovation Center for High-efficiency \\ Utilization of Solar Energy \\ Hubei University of Technology \\ China
}

\begin{abstract}
Temperature profile of an inclined elliptic cylinder is studied experimentally using optical interferometry. Interferograms of the elliptical cylinder with an isothermal surface were recorded by $\mathrm{He}-\mathrm{Ne}$ laser. The cylinder were placed with its major axis inclined $30^{\circ}$ with respect to the horizontal. Temperature profile around the cylinder was quantitatively reconstructed from the locations of fringe center. The influence of the inclination on the temperature profile around the cylinder was discussed. The results reveal that no symmetry can be found from the obtained interferogram. Optical interferometry is successfully developed to measure the temperature profile around an elliptic cylinder.
\end{abstract}

Keywords-optical interferometry; temperature; elliptic cylinder; fringe center

\section{INTRODUCTION}

Heat exchanges of horizontal elliptic cylinders have received much attention in engineering applications, including the cooling of refrigerator, space heating and oil heating. In comparison with a circular one, an elliptic cylinder provides enhanced heat transfer area at the same volume. Studies confirm that this kind of exchange enables improved heat transfer characteristics without excessive pressure drop penalty [1]. Numerical and experimental efforts have been made toward obtaining temperature and/or heat transfer around the elliptic cylinder.

Badr [2] numerically studied the flow and the thermal field around an isothermally heated, horizontal elliptic cylinder, in which the orientations varied from horizontal to vertical. Mahfouz and Kocabiyik [3] simulated the streamlines and isotherms of laminar free convection from a horizontal tube of elliptic cross-section. However, numerical approaches are based on the solution of boundary layer equation, which is inapplicable in the buoyant plume region. Akram and Hassan [4] experimentally investigated forced convection heat transfer around heated inclined cylinder. They fixed 8 thermocouples at

\author{
X. M. Cheng \\ School of Materials Science and Engineering \\ Wuhan University of Technology \\ China \\ Y.Y. Li \\ School of Materials Science and Engineering \\ Wuhan University of Technology \\ China
}

different angles to measure the surface temperature of the cylinder. This method has the incapability of full-filed measurement without disturb the flow field.

Optical interferometry based on laser has proved to be nonintrusive and full-field measurement with high precision [5,6,7] Ahmadi et al. [6] visualized the temperature profile of laminar flame jet using Mach-Zehnder interferometry (MZI). This interferometry is very sensitive to vibration and temperature change. To record interference fringes with low distortion, holographic interferometry (HI) don not require the optical components with precision as high as MZI. Chen et al. [7] investigates the effect of composition changes on temperature using HI.

In this paper, we develop temperature measurement of an inclined elliptic cylinder using holographic interferometry. The orientation was set to $30^{\circ}$. The cylinder dimensions are $350 \mathrm{~mm} \times 36 \mathrm{~mm} \times 24 \mathrm{~mm}(\mathrm{~L} \times 2 \mathrm{a} \times 2 \mathrm{~b})$. The temperature profile is reconstructed from the obtained interferogram of the cylinder. The influence concerning temperature profile around the cylinder is also discussed.

\section{THEORY}

Once light passes through the index of refractive field, the phase information of the light paths can be written in twodimensional coordinates $(\mathrm{x}, \mathrm{y})$ as

$$
\Delta \varphi=\frac{2 \pi}{\lambda}\left[n(x, y)-n_{\text {ref }}\right] L=N \pi
$$

where $\Delta \phi(\mathrm{x}, \mathrm{y})$ is the light path difference denoted by the order number of each fringe $(\mathrm{N})$. $\mathrm{L}$ is the length of the cylinder and $\lambda$ is the wavelength of laser. $\mathrm{n}(\mathrm{x}, \mathrm{y})$ and nref are the refractive index to be evaluated and in ambient, respectively. 
Applying the equation of the state of the ideal gas and the Gladstone-Dale equation [8], the temperature $\mathrm{T}(\mathrm{x}, \mathrm{y})$ can be calculated by

$$
\frac{1}{T(x, y)}=\frac{1}{T_{\text {ref }}}+\frac{2 N \lambda R \pi}{G L M P}
$$

where Tref is the ambient temperature, $\mathrm{R}$ is the universal gas constant, $\mathrm{G}$ is the Gladstone-Dale constant, $\mathrm{M}$ is the molecular weight of air and $\mathrm{P}$ is the pressure. Values of these parameters are as follows: $\mathrm{G}=2.26 \times 10-4 \mathrm{~m} 3 \cdot \mathrm{kg}-1, \mathrm{P}=1.013$ $\times 105 \mathrm{~Pa}, \mathrm{M}=28.97 \times 10-3 \mathrm{~kg} \cdot \mathrm{mol}-1$ and $\mathrm{R}=8.3143 \mathrm{~J} \cdot \mathrm{K}-$ $1 \cdot \mathrm{mol}-1$.

\section{EXPERIMENTAL SETUP}

The experimental setup of holographic interferometer is shown schematically in Fig. 1(a). The setup was conducted on a vibration isolation platform. He-Ne laser with $\lambda=632.8 \mathrm{~nm}$ wavelength was divided into the reference and object beam by a beam splitter. Each beam was reflected by mirrors and then expanded and collimated into a plane wave prior to reaching a holographic plate. An infinite fringe interferogram was focused by a lens into a CCD camera.

The elliptic cylinder was made of aluminum with major axis $2 a=36 \mathrm{~mm}$ and minor axis $2 b=24 \mathrm{~mm}$. The length of the cylinder was chosen as $350 \mathrm{~mm}$, thus the resulting flow was viewed in two-dimension. The major axis was inclined to the horizontal with an angle $\beta$, as shown in Fig. 1(b). The cylinder was heated by hot water to the uniform surface temperature $\mathrm{Ts}=335.15$. Thermocouples were placed with $100 \mathrm{~cm}$ far from the setup to measure the ambient temperature. It was indicated that the ambient temperature remained constant, Tref $=295.25 \mathrm{~K}$.
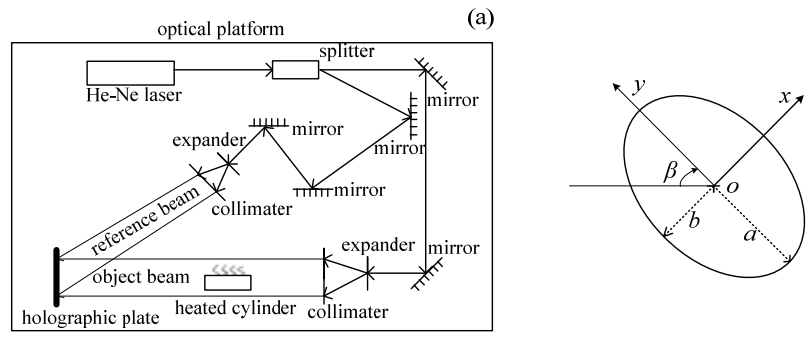

FIGURE I. FIGURE 1: (a) SKETCH OF A DOUBLE-EXPOSURE HOLOGRAPHIC INTERFEROMETER; (b) THE ELLIPTIC CYLINDER GEOMETRY

\section{RESULTS AND DISCUSSION}

The interferogram of the elliptic cylinder in air is shown in Fig. 2 while keeping the surface temperature unchanged $(\mathrm{Ts}=335.15 \mathrm{~K})$. The dimensions of the interferogram is 2384 pixel $\times 1884$ pixel and the resolution is $39.58 \mathrm{pixel} / \mathrm{mm}$. Obviously, no symmetry exists in the interferogram. The total fringe (isothermal) number is as much as 36 . Hot water flew into the cylinder through the left shadow and the other three sharp shadows were used for the position of the cylinder. Due to the buoyancy, a plume is found beyond the cylinder.

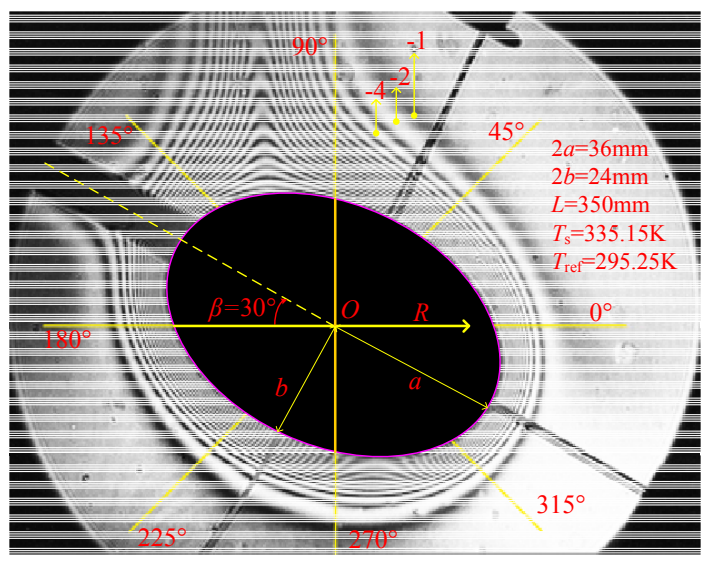

FIGURE II. THE INTERFEROGRAM AT TS $=335.15 \mathrm{~K}$ AND B=30

Fringes above the horizontal are less close than that below. They separate from each other with increase of the radial distance $(\mathrm{R})$ from the cylinder center $(\mathrm{O})$. The bright region far enough from the cylinder indicates the ambient, where the fringe was considered as the reference fringe with the order number $\mathrm{N}=0$. The phase differences of the bright and dark fringes are even and odd multiples of $\pi$ (rad), thus their order numbers were set to even and odd orders, respectively. Identifications of the order number of the fringes are presented in Fig. 2.

Although fringes are very close near the cylinder surface, a Matlab code has been developed to quickly and accurately locate the positions of the fringes centers. Because of the intensity of $\mathrm{Red}=$ Green=Blue in the grey scale image, the Red was only extracted for characteristics of the image. Sample distributions of the Red intensity and the order number at $\theta$ $=0^{\circ} \quad$ in Fig. 2 is given in Fig.3. Each maximum and minimum corresponds to a bright and dark fringe, respectively.

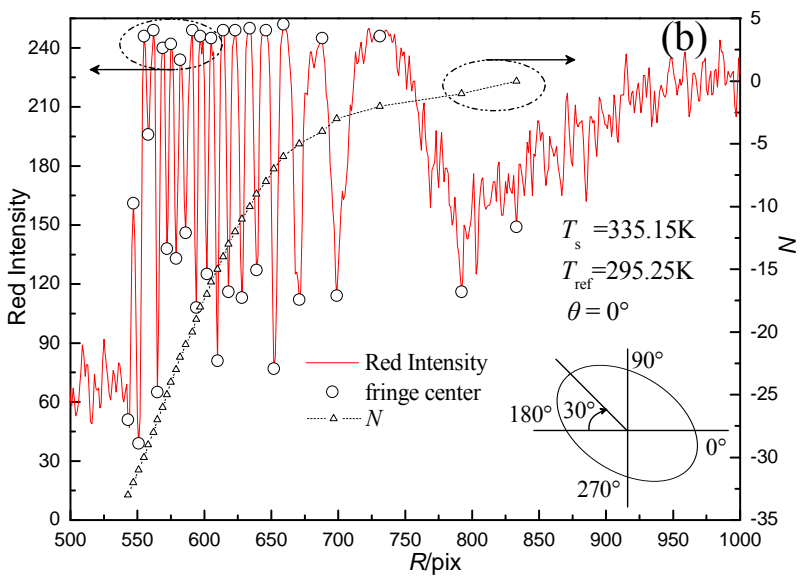

FIGURE III. RED INTENSITY VARIATION OF FRINGES IN FIG 2 AT $\Theta=0^{\circ}$

The variation of the temperature at $\theta=0^{\circ}, 45^{\circ}, 90^{\circ}$, $180^{\circ}, 225^{\circ}, 270^{\circ}, 315^{\circ}$ is shown in Fig. 4. The results can be divided into Group $1\left(\theta=45^{\circ}, 90^{\circ}, 225^{\circ}, 270^{\circ}\right)$ and Group $2\left(\theta=0^{\circ}, 135^{\circ}, 180^{\circ}, 315^{\circ}\right)$. The two groups 
correspond to the regions around the major axis and the minor axis of the elliptic cylinder, respectively.

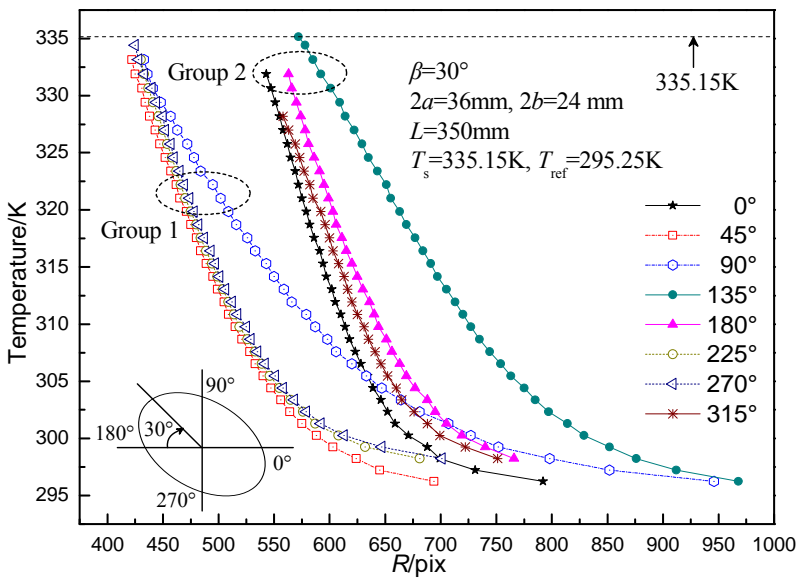

FIGURE IV. THE VARIATION OF THE TEMPERATURE AT $\Theta=0^{\circ}, 45^{\circ}, 90^{\circ}, 180^{\circ}, 225^{\circ}, 270^{\circ} 315^{\circ}$ OF THE INTERFEROGRAM IN FIG. 2.

The plume above the cylinder causes curves of the temperature at $\theta=90^{\circ}$ and $135^{\circ}$ apart from the others. Curves at $\theta=45^{\circ}, 225^{\circ}, 270^{\circ}$ show more similarity than that at $\theta=0^{\circ}, 180^{\circ}, 315^{\circ}$. The maximum temperature is evaluated at $\theta=135^{\circ}$ as $335.16 \mathrm{~K}$, in $0.008 \mathrm{~K}$ derivation with the measured one $(\mathrm{Ts}=335.15 \mathrm{~K})$, which indicates good agreement.

\section{CONCLUSION}

Temperature measurement of an inclined elliptic cylinder in air is studied with emphasis on the effects of angle of inclination. Holographic interferometry was successfully applied to obtain the interferogram of the cylinder at orientation of $30^{\circ}$. From the interference fringes, temperature profile around the cylinder is reconstructed. The results reveal that no symmetry exists in the interferogram because of the elliptic geometry. Optical interferometry based on laser can be feasible for analysis of characteristics of the temperature profile.

\section{ACKNOWLEDGEMENT}

The authors would like to gratefully acknowledge the financial support for this work received from the National Key Technology R\&D Program (Grant No. 2012BAA05B05) and the National Natural Science Foundation of China (Grant No. 51206125).

\section{REFERENCES}

[1] Rocha L. A. O., Saboya F. E. M. \& Vargas J. V. C., A comparative study of elliptical and circular section in one and two-row tubes and plate fin heat exchangers. International Journal of Heat and Fluid Flow, 18(2), pp. 247-252, 1997.

[2] Badr H. M., Laminar natural convection from an elliptic tube with different orientations. Journal of Heat Transfer, 119(4), pp. 709-718, 1997.

[3] Mahfouz F. M. \& Serpil Kocabiyik, Transient numerical simulation of buoyancy driven flow adjacent to an elliptic tube. International Journal of Heat and Fluid Flow, 24(6), pp. 864-873, 2003.
[4] Akram W. E. \& Hassan W. Z., Forced Convection Heat Transfer around Heated Inclined Cylinder. International Journal of Computer Applications, 73(8), pp. 5-11, 2013.

[5] Naylor D., Recent developments in the measurement of convective heat transfer rates by laser interferometry. International Journal of Heat and Fluid Flow, 24(3), pp. 345-355, 2003.

[6] Ahmadi M., Saffar A. M., Yousefi T., Goharkhah M., Nasr B. \& Ashiaee M., Temperature measurement of a premixed radically symmetric methane flame jet using the Mach-Zehnder interferometry. Optics and Laser in Engineering, 49(7), pp. 859-865, 2011.

[7] C. C. Chen, K. C. Chang \& S. M Tieng, Effect of composition change on temperature measurements in a premixed flame by holographic interferometry. Optical Engineering, 31(2), pp. 353-362, 1992.

[8] Bechtel J. H., Temperature measurements of the hydroxyl radical and molecular nitrogen in premixed, laminar flames by laser techniques. Applied Optics, 18(13), pp. 2100-2106, 1979. 